\title{
Identification and Heterologous Expression of the Albucidin Gene Cluster from the Marine Strain Streptomyces Albus Subsp. Chlorinus NRRL B-24108
}

\author{
Maksym Myronovskyi ${ }^{1}$, Birgit Rosenkränzer ${ }^{1}$, Marc Stierhof ${ }^{1}\left(\right.$, , Lutz Petzke ${ }^{2}$, Tobias Seiser ${ }^{2}$ \\ and Andriy Luzhetskyy $1,3, *$ \\ 1 Pharmazeutische Biotechnologie, Universität des Saarlandes, 66123 Saarbrücken, \\ Germany; maksym.myronovskyi@uni-saarland.de (M.M.); b.rosenkraenzer@mx.uni-saarland.de (B.R.); \\ s8mcstie@stud.uni-saarland.de (M.S.) \\ 2 BASF SE, 67056 Ludwigshafen, Germany; lutz.petzke@basf.com (L.P.); tobias.seiser@basf.com (T.S.) \\ 3 Helmholtz-Institut für Pharmazeutische Forschung Saarland, 66123 Saarbrücken, Germany \\ * Correspondence: a.luzhetskyy@mx.uni-saarland.de; Tel.: +49-681-302-70200
}

Received: 14 January 2020; Accepted: 7 February 2020; Published: 10 February 2020

\begin{abstract}
Herbicides with new modes of action and safer toxicological and environmental profiles are needed to manage the evolution of weeds that are resistant to commercial herbicides. The unparalleled structural diversity of natural products makes these compounds a promising source for new herbicides. In 2009, a novel nucleoside phytotoxin, albucidin, with broad activity against grass and broadleaf weeds was isolated from a strain of Streptomyces albus subsp. chlorinus NRRL B-24108. Here, we report the identification and heterologous expression of the previously uncharacterized albucidin gene cluster. Through a series of gene inactivation experiments, a minimal set of albucidin biosynthetic genes was determined. Based on gene annotation and sequence homology, a model for albucidin biosynthesis was suggested. The presented results enable the construction of producer strains for a sustainable supply of albucidin for biological activity studies.
\end{abstract}

Keywords: albucidin; herbicide; nucleoside; biosynthetic gene cluster; heterologous expression; Streptomyces albus Del14

\section{Introduction}

Pesticides play an important role in modern agriculture. Among all chemicals being produced, pesticides are in second place after fertilizers in their extent of use. A total of 2.4 billion kilograms of pesticides were applied worldwide in 2007 [1]. Nevertheless, lack of weed control is still the most topical issue. Among all pests, weeds have the largest negative effect on crop productivity [2,3]. In light of the rapidly increasing evolution of herbicide resistance, the need for new herbicides with new modes of action (MOAs) and safer ecological profiles is growing [4,5].

From all new pesticide active ingredients registered by the Environmental Protection Agency from 1997 to 2010, almost 70\% have origins in natural products. Interestingly, only 8\% of conventional herbicides are natural product-derived [3,6]. The wide structural diversity of natural products and their small amount of overlap with synthetic compounds imply their potential as lead structures for the development of new pesticides [7-9]. This is further confirmed by the phytotoxin literature, which suggests that natural products have many more MOAs than the commercial herbicides currently possess [3].

A novel bleaching herbicide, albucidin, from the strain Streptomyces albus subsp. chlorinus NRRL B-24108 was discovered in 2009 [10]. In this paper, we present the identification, heterologous 
expression, and engineering of the albucidin gene cluster. We also propose the biosynthetic route that leads to the production of albucidin. The identified minimal set of biosynthetic genes allows for the straightforward construction of overproducing strains for a high yield albucidin supply for biological activity studies.

\section{Materials and Methods}

\subsection{General Experimental Procedures}

All strains, plasmids and BACs used in this work are listed in Tables S1 and S2. Escherichia coli strains were cultured in LB medium [11]. Streptomyces strains were grown on soya flour mannitol agar (MS agar) [12] and in liquid tryptic soy broth (TSB; Sigma-Aldrich, St. Louis, MO, USA). For albucidin production, liquid SG medium [13] was used. The antibiotics kanamycin, apramycin, hygromycin, ampicillin and nalidixic acid were supplemented when required.

\subsection{Isolation and Manipulation of DNA}

The previously constructed BAC library of Streptomyces albus subsp. chlorinus NRRL B-24108 was used [14]. DNA manipulation, E. coli transformation and E. coli/Streptomyces intergeneric conjugation were performed according to standard protocols $[11,12,15]$. BAC DNA was purified with the BACMAX ${ }^{\mathrm{TM}}$ DNA purification kit (Lucigen, Middleton, WI, USA). Restriction endonucleases were used according to the manufacturer's recommendations (New England Biolabs, Ipswich, MA, USA). All the strains and plasmids are listed in the Tables S1 and S2, respectively.

\subsection{Metabolite Extraction and Analysis}

For metabolite extraction, Streptomyces strains were grown in $15 \mathrm{~mL}$ of TSB in a $100 \mathrm{~mL}$ baffled flask for 1 day, and $1 \mathrm{~mL}$ of seed culture was used to inoculate $100 \mathrm{~mL}$ of SG production medium in a $500 \mathrm{~mL}$ baffled flask. Cultures were grown for 7 days at $28^{\circ} \mathrm{C}$ and $180 \mathrm{rpm}$ in an Infors multitron shaker. Albucidin was extracted from the culture supernatant with an equal amount of butanol, evaporated, and dissolved in methanol. Albucidin production was analysed on a Bruker Amazon Speed mass spectrometer coupled to UPLC Thermo Dionex Ultimate 3000 RS. Analytes were separated either on a Waters ACQUITY BEH C18 column $(1.7 \mu \mathrm{m}, 2.1 \mathrm{~mm} \times 30 \mathrm{~mm})$ or on a Waters ACQUITY BEH C18 column $(1.7 \mu \mathrm{m}, 2.1 \mathrm{~mm} \times 100 \mathrm{~mm})$. Water $+0.1 \%$ formic acid and methanol $+0.1 \%$ formic acid were used as the mobile phases. For the determination of high-resolution mass, analytes were analysed with a Thermo LTQ Orbitrap XL coupled to UPLC Thermo Dionex Ultimate 3000 RS. Analytes were separated on a Waters ACQUITY BEH C18 column $(1.7 \mu \mathrm{m}, 2.1 \mathrm{~mm} \times 100 \mathrm{~mm})$ with water $+0.1 \%$ formic acid and methanol $+0.1 \%$ formic acid as the mobile phase.

\subsection{Chemical Mutagenesis}

One millilitre of spore suspension of Streptomyces albus subsp. chlorinus NRRL B-24108 was inoculated into $100 \mathrm{~mL}$ of SG medium in a $500 \mathrm{~mL}$ baffled flask and cultivated overnight at $28^{\circ} \mathrm{C}$ and $180 \mathrm{rpm}$. The $\mathrm{pH}$ of the culture was adjusted to 8.5 with $1 \mathrm{M} \mathrm{NaOH}$. Ten millilitres of culture was transferred into three $50 \mathrm{~mL}$ falcon tubes. Then, $64 \mathrm{mg}$ of wet NTG was dissolved in $16 \mathrm{~mL}$ of water. Next, $6.666 \mathrm{~mL}, 4.285 \mathrm{~mL}$ and $1.765 \mathrm{~mL}$ of NTG stock solution were added to the tubes containing culture to reach final NTG concentrations of $800 \mu \mathrm{g} / \mathrm{mL}, 600 \mu \mathrm{g} / \mathrm{mL}$ and $300 \mu \mathrm{g} / \mathrm{mL}$, respectively. The samples were incubated at $28^{\circ} \mathrm{C}$ for $30 \mathrm{~min}$ in the overhead shaker. The mycelium was precipitated by centrifugation, and the supernatant was discarded. The mycelium was washed twice with $5 \%$ sodium thiosulfate solution. The treated samples were plated on MS agar plates and cultivated for 14 days at $28^{\circ} \mathrm{C}$. The spores were washed with water and plated in dilutions on MS agar. The plates with spore dilutions were incubated for 10 days at $28^{\circ} \mathrm{C}$. Single colonies were picked on $30 \mathrm{~mm}$ plates with SG agar. The plates were incubated for 14 days at $28^{\circ} \mathrm{C}$. Agar blocks were cut out from the plates and 
transferred into $2 \mathrm{~mL}$ tubes. Albucidin was extracted from the agar blocks with $500 \mu \mathrm{L}$ of butanol for $48 \mathrm{~h}$. The extracts were analysed using HPLC-MS.

\subsection{Albucidin Isolation and ${ }^{1} H-N M R$ Spectroscopy}

Streptomyces albus $1 \mathrm{~K} 1$ was grown in $10 \mathrm{~L}$ of SG medium, and albucidin was extracted with butanol. The dry extract was dissolved in $50 \mathrm{~mL}$ of water containing $5 \%$ acetonitrile and $0.1 \%$ formic acid. The extract was loaded onto 3 C18 SPE columns (Discovery DSC-18 SPE 52607-U) equilibrated with $5 \%$ acetonitrile in water with $0.1 \%$ formic acid. The flowthrough was collected. The columns were washed twice with $12 \mathrm{~mL}$ of $5 \%$ acetonitrile containing $0.1 \%$ formic acid. The flowthrough and the wash fractions were combined and evaporated in a rotary evaporator. The presence of albucidin was detected by HPLC-MS.

The dry material after the SPE purification step was dissolved in methanol and used for size-exclusion chromatography. Separation was performed on a glass column $(30 \mathrm{~mm} \times 1000 \mathrm{~mm})$ packed with Sephadex LH-20 and methanol as the mobile phase. Fractions containing albucidin were identified by HPLC-MS. Albucidin-containing fractions were combined and evaporated. The dry extract was dissolved in $5 \mathrm{~mL}$ of $5 \%$ methanol in water containing $10 \mathrm{mM}$ potassium phosphate buffer pH 6.4.

HPLC separation was performed on a preparative HPLC Thermo Dionex Ultimate 3000 equipped with a Macherey Nagel Nucleodur HTec C18 column $(5 \mu \mathrm{m}, 21 \mathrm{~mm} \times 150 \mathrm{~mm})$. A $10 \mathrm{mM}$ potassium phosphate buffer ( $\mathrm{pH}$ 6.4) was used as solvent $\mathrm{A}$, and $50 \%$ methanol in $10 \mathrm{mM}$ phosphate buffer $(\mathrm{pH}$ $6,4)$ was used as solvent $B$. The following gradient at a flowrate of $15 \mathrm{~mL} / \mathrm{min}$ was used for separation: 0 min $-13 \%$ B, 20 min $-25 \%$ B, 21 min- $25 \%$ B, 24 min-100\% B, 25 min- $100 \%$ B, 28 min- $13 \%$ B, 29 min- $13 \%$ B. Albucidin eluted at $18 \mathrm{~min}$. The albucidin-containing fractions were pooled and evaporated.

For the final purification step, the dry material after HPLC purification was dissolved in $5 \mathrm{~mL}$ of water and loaded onto a Sephadex LH-20 column $(30 \mathrm{~mm} \times 450 \mathrm{~mm})$ previously equilibrated with water. Water was used as the mobile phase. The fractions containing albucidin were identified by HPLC-MS, pooled and evaporated.

The ${ }^{1} \mathrm{H}-\mathrm{NMR}$ spectra were recorded on a Bruker Avance 500 spectrometer (Bruker, BioSpin GmbH, Rheinstetten, Germany) at $300 \mathrm{~K}$ equipped with a $5 \mathrm{~mm}$ BBO probe using deuterated trifluoroacetic acid (Deutero, Kastellaun, Germany) as the solvent containing tetramethylsilane (TMS) as a reference. Albucidin was measured in deuterated water (Deutero, Kastellaun, Germany). The chemical shifts are reported in parts per million (ppm) relative to TMS. All spectra were recorded with the standard ${ }^{1} \mathrm{H}$ pulse program using 128 scans. The structure of albucidin was confirmed by comparison of the recorded ${ }^{1} \mathrm{H}$ NMR data (Figure S1) with published data [10].

\subsection{Construction of the $1 K 1$ BAC Derivatives}

The derivatives of 1K1 BAC with gene deletions were constructed using the RedET approach. For this, the antibiotic resistance marker was amplified by PCR with primers harbouring overhang regions complementary to the boundaries of the DNA to be deleted. The amplified fragment was used for recombineering of the BAC. The recombinant BACs were analysed by restriction mapping and sequencing. The primers used for recombineering purposes are listed in Table S3.

For the construction of BAC 1K1_LS, the ampicillin marker from pUC19 was amplified with the primers LS-F/LS-R. For the construction of the BAC 1K1_RS, the hygromycin marker from pACS-hyg [16] was amplified with the primers RS-F/RS-R. For the construction of the BACs 1K1_KO14, 1K1_KO15 and 1K1_KO16, the ampicillin cassette was amplified with the pairs of primers KO14-F/KO14-R, KO15-F/KO15-R and KO16-F/KO16-R, respectively. For the construction of the BACs 1K1_KO7, 1K1_KO8, 1K1_KO9, 1K1_KO10, 1K1_KO11, 1K1_KO12 and 1K1_KO13, the ampicillin cassette was amplified with the pairs of primers KO7-F/KO7-R, KO8-F/KO8-R, KO9-F/KO9-R, KO10-F/KO10-R, KO11-F/KO11-R, KO12-F/KO12-R and KO13-F/KO13-R, respectively. 
BAC 1K1_alb_act was constructed in two steps. First, 1K1_RS2 BAC was constructed from $1 \mathrm{~K} 1$ using an ampicillin marker amplified with primers RS2-F/RS2-R. Then BAC 1K1_alb_act was constructed by recombineering the BAC 1K1_RS2 using a hygromycin marker from pACS-hyg amplified with the primers ACT-F/ACT-R.

\subsection{Genome Mining and Bioinformatics Analysis}

The S. albus subsp. chlorinus genome was screened for secondary metabolite biosynthetic gene clusters using the antiSMASH online tool [17] and the software Geneious [18]. The genomic sequence of the albucidin producer S. albus subsp. chlorinus NRRL B-24108 was deposited in GenBank under accession number VJOK00000000 [14].

\section{Results and Discussion}

\subsection{Identification of the Albucidin Biosynthetic Gene Cluster}

The aim of this study was to identify the biosynthetic genes leading to the production of the nucleoside phytotoxin albucidin. For this purpose, the genome sequence of the producer strain of Streptomyces albus subsp. chlorinus NRRL B-24108 was analysed by genome-mining software [17]. This analysis led to the identification of several putative nucleoside clusters. To prove the involvement of these candidate clusters in albucidin production, they were heterologously expressed in a genetically engineered cluster-free strain Streptomyces albus Del14 [19] and in Streptomyces lividans TK24 [20]. No albucidin production was detected in the extracts of the obtained strains, indicating that either the expressed clusters were not involved in the biosynthesis of albucidin or they were not expressed in the heterologous host environment. The inactivation of the candidate clusters in the natural albucidin producer was not feasible because the strain is refractory to genetic manipulation. Considering the difficulties in identifying the albucidin gene cluster using conventional methods, an alternative approach using chemical mutagenesis was chosen.

For chemical mutagenesis of the albucidin-producing strain S. albus subsp. chlorinus NRRL B-24108, 1-methyl-3-nitro-1-nitrosoguanidine (NTG) was used. The strain in the exponential growth stage was treated with various NTG concentrations $(800 \mu \mathrm{g} / \mathrm{mL}, 600 \mu \mathrm{g} / \mathrm{mL}$ and $300 \mu \mathrm{g} / \mathrm{mL})$ for $30 \mathrm{~min}$. After mutagenesis, the cells were washed with 5\% thiosulfate solution and plated in dilutions on MS-agar medium for segregation of mutations. The spores of the obtained mutant populations were washed and plated on MS-agar plates in dilutions to obtain single colonies. Altogether, 4000 individual mutants were analysed for albucidin production. The mutants were cultivated on individual plates with the production medium SG agar. The metabolites were extracted with butanol, and albucidin production was assayed by HPLC-MS. Eight mutants that lost the ability to produce albucidin were identified in the course of this screening: 6-238, 6-260, 6-389, 6-444, 6-612, 6-892, 8-610 and 8-639. The genomic DNA of the obtained zero mutants was sequenced using Illumina technology. The point mutations in the genomes of the mutants were detected by mapping the sequencing reads to the reference genome of the wild type albucidin producer. Up to 100 transition mutations were identified in the genomes of the mutant strains. By comparing the mutation patterns of the separate mutants, a short genomic region was identified that was affected by point mutations in all analysed zero mutants, implying its potential involvement in albucidin production (Figure S2). The identified region contains two genes, SACHL2_05525 and SACHL2_05524, which encode putative radical SAM proteins and were named albA and albB (Table 1, Figure $1 \mathrm{~b}$ ). The genes constitute a putative operon with the third gene SACHL2_05523, which was named albC. The albC gene encodes a putative ribonucleoside-triphosphate reductase and was not affected by point mutations in the analysed zero mutants of $S$. albus subsp. chlorinus NRRL B-24108. The identified genes albA and albB were not a part of the nucleoside gene clusters previously identified by genome mining and analysed in this study. Interestingly, these genes were located within the DNA fragment annotated by genome mining software as a putative NRPS gene cluster. 
Table 1. Genes encoded within the chromosomal fragment cloned in BAC 1K1.

\begin{tabular}{|c|c|c|}
\hline Gene & Locus Tag 1 & Putative Function \\
\hline 1 & SACHL2_05539 & Hypothetical protein \\
\hline 2 & SACHL2_05538 & $\mathrm{ABC}$ transporter \\
\hline 3 & SACHL2_05537 & Transcriptional regulatory protein LiaR \\
\hline 4 & SACHL2_05536 & Hypothetical protein \\
\hline 5 & SACHL2_05535 & Hypothetical protein \\
\hline 6 & SACHL2_05534 & beta-lactamase/D-alanine carboxypeptidase \\
\hline 7 & SACHL2_05533 & Chondramide synthase \\
\hline 8 & SACHL2_05532 & Hypothetical protein \\
\hline 9 & SACHL2_05531 & Hypothetical protein \\
\hline 10 & SACHL2_05530 & Thymidylate kinase \\
\hline 11 & SACHL2_05529 & Pyruvate, phosphate dikinase \\
\hline 12 & SACHL2_05528 & Hypothetical protein \\
\hline 13 & SACHL2_05527 & Hypothetical protein \\
\hline 14 & SACHL2_05526 & Hypothetical protein \\
\hline $15 ;$ albA & SACHL2_05525 & Biotin synthase, radical SAM protein \\
\hline $16 ; a l b B$ & SACHL2_05524 & Radical SAM protein \\
\hline $17 ; a l b C$ & SACHL2_05523 & Ribonucleoside-triphosphate reductase \\
\hline 18 & SACHL2_05522 & Tyrocidine synthase 3 \\
\hline 19 & SACHL2_05521 & Plipastatin synthase, subunit A \\
\hline 20 & SACHL2_05520 & Acyl carrier protein \\
\hline 21 & SACHL2_05519 & Demethylmenaquinone methyltransferase \\
\hline 22 & SACHL2_05518 & Linear gramicidin synthase, subunit D \\
\hline 23 & SACHL2_05517 & Hypothetical protein \\
\hline 24 & SACHL2_05516 & Acyl carrier protein \\
\hline 25 & SACHL2_05515 & Fatty-acid-CoA ligase \\
\hline 26 & SACHL2_05514 & Ribonucleotide-diphosphate reductase \\
\hline 27 & SACHL2_05513 & Hypothetical protein \\
\hline 28 & SACHL2_05512 & Tyrocidine synthase 3 \\
\hline 29 & SACHL2_05511 & Hypothetical protein \\
\hline
\end{tabular}

${ }^{1}$ The locus tags refer to the genome sequence of S. albus subsp. chlorinus NRRL B-24108 available under GenBank accession number VJOK00000000. 
(a) $1 \mathrm{~K} 1$

2D4

(b)

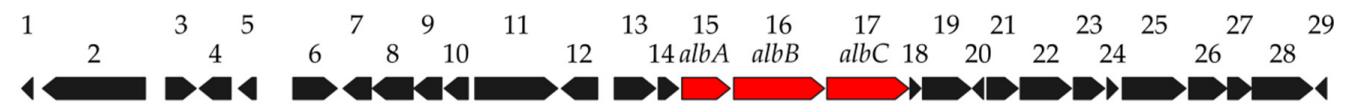

(c)

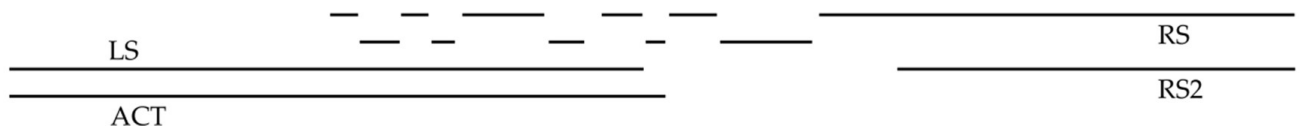

Figure 1. Chromosomal fragment of S. albus subsp. chlorinus NRRL B-24108 with the albucidin biosynthetic genes. (a) Schematic representations of DNA fragments cloned in BACs 1K1 and 2D4; (b) The genes encoded within the fragment cloned in BAC $1 \mathrm{~K} 1$. The albA-C operon is marked in red; and (c) Overview of the performed deletions within BAC 1K1.

To determine whether the identified genes $a l b A$ and $a l b B$ encode albucidin biosynthetic enzymes, a BAC $1 \mathrm{~K} 1$ containing the abovementioned genes (Figure 1a) was selected from the genomic library of S. albus subsp. chlorinus NRRL B-24108. BAC 1K1 was transferred into the heterologous host strains S. albus Del14 and S. lividans TK24 by conjugation, and the production profile of the obtained strains S. albus $1 \mathrm{~K} 1$ and S. lividans $1 \mathrm{~K} 1$ was analysed by HPLC-MS. The production of the compound with a high-resolution mass corresponding to albucidin could be detected in the extracts of S. albus $1 \mathrm{~K} 1$ (Figure S3). No production could be detected in S. lividans 1K1.

Due to the lack of an albucidin standard, we set out to purify the compound identified in the extracts of $S$. albus $1 \mathrm{~K} 1$ for structure elucidation studies by NMR spectroscopy. The S. albus $1 \mathrm{~K} 1$ strain was cultivated in $10 \mathrm{~L}$ of SG medium for 7 days. The culture supernatant was extracted with equal amount of butanol, and the obtained extract was concentrated under vacuum. Four milligrams of the compound was purified using size exclusion and reverse phase chromatography and used for subsequent NMR studies. Analysis of the recorded NMR spectra of the purified compound unequivocally demonstrated its identity as albucidin (Figure S1 and Figure 2a).<smiles></smiles>

(a)

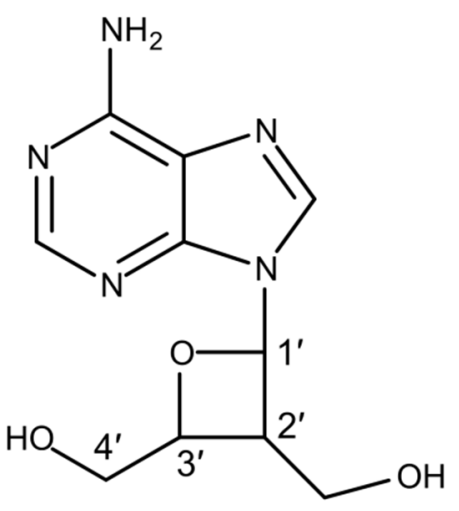

(b)

Figure 2. The structures of (a) albucidin and (b) oxetanocin A.

The production of albucidin by $S$. albus $1 \mathrm{~K} 1$ gives evidence that the genes albA and albB identified by chemical mutagenesis encode albucidin biosynthetic genes. The lack of albucidin production by $S$. lividans $1 \mathrm{~K} 1$ can be explained by differences in regulatory networks of the S. albus Del14 and S. lividans TK24 strains. 


\subsection{Identification of the Minimal Set of Albucidin Biosynthetic Genes}

BAC $1 K 1$, which leads to the production of albucidin under expression in the heterologous host S. albus Del14, contains a $32 \mathrm{~kb}$ chromosomal fragment from the natural albucidin producer S. albus subsp. chlorinus NRRL B-24108. Twenty-nine open reading frames were annotated in this $32 \mathrm{~kb}$ region (Table 1, Figure 1b). Of these genes, only two, alb $A$ and $a l b B$, were affected by point mutations in albucidin zero mutants identified in the course of the chemical mutagenesis studies. These two genes constitute a putative operon with the gene albC, implying that either only the genes alb $A$ and $a l b B$ are necessary for albucidin production or that all three gene within the operon are required. To experimentally determine the minimal set of albucidin biosynthetic genes, a series of gene deletions was performed within the cloned region of $1 \mathrm{~K} 1 \mathrm{BAC}$.

The genes albA-C are located in the middle part of the chromosomal fragment cloned in 1K1 BAC. The alb operon is preceded by the genes SACHL2_05539-SACHL2_05526, followed by the genes SACHL2_05522-SACHL2_05511 (Table 1). For the sake of simplicity, the 29 genes SACHL2_05539-SACHL2_05511 cloned in the BAC 1K1 will be designated in the text according to their sequence number ( 1 to 29 ). (Figure $1 \mathrm{~b}$ ) To determine which genes within the $1 \mathrm{~K} 1$ cloned fragment are essential for albucidin production, five deletions (LS, KO14, KO15, $\mathrm{KO} 16$ and RS) were performed in the 1K1 BAC yielding the BACs 1K1_LS, 1K1_KO14, 1K1_KO15, 1K1_KO16 and 1K1_RS (Figure 1c). In BAC 1K1_LS, the left shoulder encompassing genes 1-13 was substituted by an ampicillin resistance marker (Figure 1c). The genes 14, albA (gene 15) and albB (gene 16) were substituted with the ampicillin resistance gene in BACs 1K1_KO14, 1K1_KO15 and 1K1_KO16, respectively (Figure 1c). In the BAC 1K1_RS, the right shoulder encompassing genes albC (gene 17)-28 was substituted by the hygromycin resistance marker (Figure 1c). The constructed BACs were transferred separately into the S. albus Del14 strain by conjugation, and the albucidin production of the resulting strains was assayed by HPLC-MS.

The deletion of gene 14 did not affect albucidin production in S. albus 1K1_KO14 (Figure S4B). As expected from the results of chemical mutagenesis, inactivation of the genes albA (gene 15) and albB (gene 16) completely abolished albucidin production in the strains S. albus 1K1_KO15 and S. albus 1K1_KO16 (Figure S4C,D). This unambiguously demonstrates the essential role of the genes albA and albB in albucidin biosynthesis.

Deletion of the genes albC (gene 17)-28 did not affect albucidin production by the S. albus RS strain (Figure S4F). It was expected from the gene annotation and results of chemical mutagenesis that the genes 18-28 do not participate in albucidin biosynthesis. However, the dispensability of the gene albC (gene 17) is surprising since it belongs to the same operon as the essential genes albA (gene 15) and albB (gene 16). The deletion of the albC gene (gene 17) might be cross-complemented by an unidentified gene in the genome of the host strain S. albus Del14.

Albucidin production was heavily abolished in the strain S. albus 1K1_LS (Figure S4E), implying that at least one of the genes 1-13 that were deleted in the BAC 1K1_LS might be essential for albucidin biosynthesis. No genes encoding regulatory proteins or structural enzymes that might participate in nucleoside biosynthesis were identified in close proximity to the albA-C operon. To identify the genes within the deleted LS region that influence albucidin production, a BAC 2D4 was isolated from the genomic library of $S$. albus subsp. chlorinus NRRL B-24108. The chromosomal fragment cloned in BAC 2D4 overlaps with the fragment cloned in BAC $1 \mathrm{~K} 1$ and covers the albA-C operon (Figure 1a). In contrast to 1K1, 2D4 BAC lacks genes 1-6, which are present in the deleted LS region. BAC 2D4 was transferred into S. albus Del14. Albucidin production could be detected in the extracts of the obtained strain S. albus 2D4 by HPLC-MS (Figure S5). This indicates that the genes 1-6 within the LS region are not involved in albucidin production and that one of the genes among 7-13 is responsible for the abolishment of albucidin production in S. albus 1K1_LS.

To identify which of the genes 7-13 is involved in albucidin biosynthesis, each of them was individually substituted by an ampicillin resistance marker in 1K1 BAC yielding 1K1_KO7, 1K1_KO8, 1K1_KO9, 1K1_KO10,1K1_KO11, 1K1_KO12 and 1K1_KO13 (Figure 1b,c). The constructed BACs were transferred into the $S$. albus Del14 strain, and the albucidin production was analysed. The albucidin 
production levels of all obtained strains, except S. albus 1K1_KO12, were in the range of S. albus $1 \mathrm{~K} 1$ harbouring the unmodified BAC (Figure S6). Albucidin production was abolished in S. albus 1K1_KO12 (Figure S6G), indicating that gene 12 is responsible for the detrimental effect of the LS deletion on albucidin biosynthesis. No enzymatic activity could be assigned to the peptide product of gene 12 using blast analysis. The product also did not show homology to any known regulatory protein. Considering this, it was proposed that only the genes albA and albB encode structural enzymes essential for albucidin production in the heterologous host $S$. albus Del14 and that the product of the gene 12 elicits a regulatory effect on transcription of the albA-C operon through a mechanism that is not understood. To prove this, a BAC $1 \mathrm{~K} 1$ _alb_act was constructed containing only albA-C genes under the control of a strong promoter. The genes downstream of the albA-C operon (genes 18-28) were substituted with the ampicillin resistance gene and the genes upstream of the operon (genes 1-14) were substituted with the hygromycin resistance gene (Figure 1c). The hygromycin resistance gene used was under the control of the strong synthetic promoter TS81 and did not contain a terminator at its 3'-end [21]. The insertion of the hygromycin marker in front of the albA-C genes was performed in the orientation, which enabled their read-through from the TS81 promoter and their transcriptional activation. The constructed BAC 1K1_alb_act was transferred into the heterologous host strain $S$. albus Del14. The production of albucidin was detected in the extracts of the obtained strain S. albus 1K1_alb_act by HPLC-MS (Figure S7). Three times increase of albucidin production was observed in the strain S. albus 1K1_alb_act compared to $S$. albus $1 \mathrm{~K} 1$ containing non-modified albucidin cluster (Figure S7). Taking into account that the total recovered albucidin yield from the S. albus $1 \mathrm{~K} 1$ strain was approximately $0.4 \mathrm{mg} / \mathrm{L}$, the calculated albucidin production by $S$. albus $1 \mathrm{~K} 1$ _alb_act corresponded to $1.2 \mathrm{mg} / \mathrm{L}$. The albucidin production rate of $2 \mathrm{mg} / \mathrm{L}$ was reported for the original producer Streptomyces albus subsp. chlorinus NRRL B-24108 [10].

Albucidin production by the strain S. albus $1 \mathrm{~K} 1 \_$alb_act clearly demonstrates that the genes albA and $a l b B$ constitute the minimal set of the genes required for albucidin biosynthesis in heterologous host $S$. albus Del14. The role of the gene albC in albucidin biosynthesis is not completely understood. Because $a l b C$ constitutes a single operon with $a l b A$ and $a l b B$ and its product shows homology to nucleotide biosynthetic enzymes, it cannot be completely excluded that the albC is involved in albucidin production in the natural producer. However, the deletion of $a l b C$ has no effect on albucidin production in heterologous host.

The identification of the minimal set of albucidin biosynthetic genes allows its expression in various heterologous chassis strains as well as rational construction of albucidin overproducers. The engineering of the albucidin biosynthetic genes can be performed in E. coli and the obtained constructs can be heterologously expressed in Streptomyces hosts. In contrast to the genetically intractable original albucidin producer Streptomyces albus subsp. chlorinus NRRL B-24108, commonly used heterologous strains possess a well-established toolkit for their genetic manipulation. This opens the possibility to engineer their metabolic network to increase the intracellular levels of biosynthetic precursors and therefore to increase the production yields. The heterologous strains are often characterized by the simplified metabolic background which provides better detection limits for heterologously expressed compounds than the original producers, higher product yields and simplified downstream processing. Construction of the albucidin overproducers based on heterologous expression hosts is not necessarily limited to a rational approach. The chassis strains expressing heterologous cluster may be also subjected to classical mutagenesis and screening for overproducing clones.

\subsection{Proposed Biosynthetic Pathway of Albucidin}

Structurally, albucidin is closely related to oxetanocin A (Figure $2 b$ ), which has been isolated from the culture of Bacillus megaterium NK84-0218 [22]. Both compounds are the only known naturally occurring nucleosides featuring four membered oxetane rings in their structure. From a structural view, albucidin is 2 -dehydroxymethyl oxetanocin A. Two genes, $0 x s A$ and $o x s B$, encoding a putative HD domain phosphohydrolase and a cobalamin-dependent S-adenosylmethionine radical enzyme 
have been reported to be responsible for oxetanocin biosynthesis [23]. dAMP, dADP and dATP were identified as direct oxetanocin precursors [24]. The product of oxs $B$ catalyses the contraction of the deoxyribose ring, while the product of $o x s A$ is responsible for the removal of one or multiple phosphates from a phosphorylated $2^{\prime}$-deoxyadenosine derivative [24,25]. Through the simultaneous actions of OxsA and OxsB, the phosphorylated 2'-deoxyadenosine is converted to the oxetanocin A precursor, its aldehyde form, which must be reduced to complete biosynthesis [24]. This reaction is not encoded by the genes within the oxetanocin A cluster and is likely to be carried by an unidentified enzyme of $B$. megaterium NK84-0218.

Gene inactivation studies have given evidence that two genes, albA and albB, are required for the production of albucidin. Both genes encode putative SAM radical proteins. At the protein level, the albA gene shows homology to biotin synthases and the albB gene shows homology to the product of the oxetanocin biosynthetic gene oxs $B$. Despite the high structural similarity of albucidin and oxetanocin $\mathrm{A}$, the homologue of the second oxetanocin biosynthetic gene oxs $A$ cannot be found within the albucidin cluster or in the genome of albucidin producer S. albus subsp. chlorinus NRRL B-24108. The homology of the albB gene to oxs $B$ implies that the product of albB might also be responsible for the ring contraction reaction in albucidin biosynthesis. However, the structural differences between albucidin and oxetanocin and the absence of an oxs $A$ homologue imply substantial differences in biosynthetic routes leading to the biosynthesis of the nucleosides. Due to the lack of an oxs $A$ homologue that is responsible for the dephosphorylation of adenine deoxyribonucleotides during oxetanocin biosynthesis, we propose that deoxyadenosine is used instead of dAMP, dADP or dATP as a precursor for albucidin production. The product of $a l b B$ is likely responsible for the contraction of the deoxyribose ring of deoxyadenosine (Figure 3) in a similar manner as its homologue OxsB catalyses oxetane ring formation in oxetanocin A biosynthesis [24]. As a result of this reaction, the aldehyde form of oxetanocine $\mathrm{A}$ is formed. The conversion of the latter into albucidin is likely to be catalysed by the product of albA, which removes the aldehyde group from the 2'-position (Figure 3).

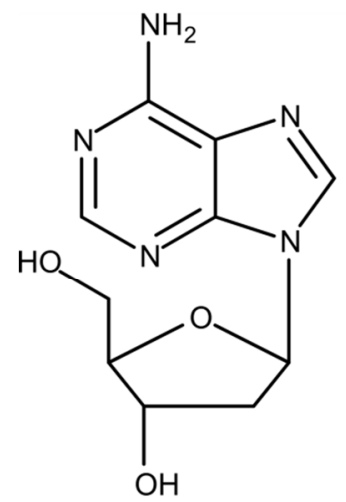

1

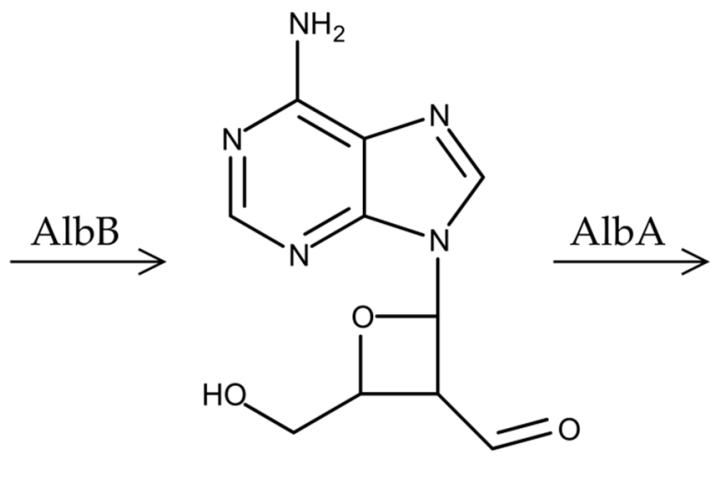

2

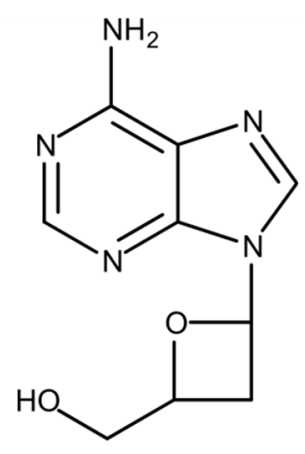

3

Figure 3. The proposed scheme of albucidin biosynthesis. $2^{\prime}$-Deoxyadenosine (1) is converted into the aldehyde form of oxetanocin A (2) by the product of $a l b B$. The latter is then converted into albucidin (3) by the product of albA.

In this paper, we report the identification, cloning, and heterologous expression of the albucidin biosynthetic gene cluster from Streptomyces albus subsp. chlorinus NRRL B-24108. Albucidin is a nucleoside phytotoxin featuring a rare oxetane ring in its structure. This metabolite shows herbicidal activity against a broad spectrum of grass and broadleaf weeds. In treated plants, albucidin induces metabolic perturbation, chlorosis, and bleaching [10]. The exact MOA of the compound remains unknown. The identification of the albucidin cluster presented in this paper enables biosynthetic studies of albucidin, optimization of its production as well as albucidin supply for the determination of its MOA. 


\section{Patents}

(WO2018224939) Gene cluster for the biosynthesis of albucidin.

Supplementary Materials: The following are available online at http://www.mdpi.com/2076-2607/8/2/237/s1, Figure S1: ${ }^{1} \mathrm{H}$ NMR spectrum of albucidin (500 MHz), Figure S2: The genes albA and albB with the mapped point mutations identified in the course of NTG-mutagenesis, Figure S3: High resolution HPLC-MS analysis of albucidin production by Streptomyces albus 1K1, Figure S4: HPLC-MS analysis of albucidin production by Streptomyces albus strain harboring 1K1 BAC and its derivatives with gene deletions, Figure S5: HPLC-MS analysis of albucidin production by Streptomyces albus 1K1 BAC (a) and Streptomyces albus 2D4 BAC (b), Figure S6: HPLC-MS analysis of albucidin production by Streptomyces albus strain harboring $1 \mathrm{~K} 1 \mathrm{BAC}$ and its derivatives with gene deletions, Figure S7: HPLC-MS analysis of albucidin production by Streptomyces albus strain harboring full length 1K1 BAC (a) and minimized 1K1_alb_act BAC, containing only transcriptionally activated albA-C operon (b), Table S1: Bacterial strains used in the study, Table S2: Plasmids and BACs used in the study, Table S3: Primers used in this study. References $[26,27]$ are cite $d$ in the supplementary materials.

Author Contributions: L.P., T.S., M.M., and A.L. designed the experiments; M.M. and B.R. performed the experiments; M.S. performed structure elucidation studies; M.M., B.R., and A.L. analysed the data and wrote the manuscript, and all authors reviewed the manuscript. All authors have read and agreed to the published version of the manuscript.

Funding: This work was partially supported by the BMBF grant "MyBio" 031B0344B.

Conflicts of Interest: The authors declare no conflict of interest.

\section{References}

1. Malakof, D.; Stokstad, E. Infographic: Pesticide Planet. Science 2013, 341, 730-731.

2. Pimentel, D.; Zuniga, R.; Morrison, D. Update on the environmental and economic costs associated with alien-invasive species in the United States. Ecol. Econ. 2005, 52, 273-288. [CrossRef]

3. Dayan, F.E.; Duke, S.O. Natural compounds as next-generation herbicides. Plant Physiol. 2014, 166, 1090-1105. [CrossRef]

4. Duke, S.O. Why have no new herbicide modes of action appeared in recent years? Pest Manag. Sci. 2012, 68, 505-512. [CrossRef]

5. Heap, I. Global perspective of herbicide-resistant weeds. Pest Manag. Sci. 2014, 70, 1306-1315. [CrossRef]

6. Cantrell, C.L.; Dayan, F.E.; Duke, S.O. Natural products as sources for new pesticides. J. Nat. Prod. 2012, 75, 1231-1242. [CrossRef]

7. Duke, S.O.; Stidham, M.A.; Dayan, F.E. A novel genomic approach to herbicide and herbicide mode of action discovery. Pest Manag. Sci. 2019, 75, 314-317. [CrossRef]

8. Harvey, A.L. Natural products as a screening resource. Curr. Opin. Chem. Biol. 2007, 11, 480-484. [CrossRef]

9. Koch, M.A.; Schuffenhauer, A.; Scheck, M.; Wetzel, S.; Casaulta, M.; Odermatt, A.; Ertl, P.; Waldmann, H. Charting biologically relevant chemical space: A structural classification of natural products (SCONP). Proc. Natl. Acad. Sci. USA 2005, 102, 17272-17277. [CrossRef]

10. Hahn, D.R.; Graupner, P.R.; Chapin, E.; Gray, J.; Heim, D.; Gilbert, J.R.; Gerwick, B.C. Albucidin: A novel bleaching herbicide from Streptomyces albus subsp. chlorinus NRRL B-24108. J. Antibiot. 2009, 62, 191-194. [CrossRef]

11. Green, M.R.; Sambrook, J. Molecular Cloning: A Laboratory Manual, 4th ed.; Cold Spring Harbor Laboratory Press: New York, NY, USA, 2012.

12. Kieser, T.; Bibb, M.J.; Buttner, M.J.; Chater, K.F.; Hopwood, D.A. Practical Streptomyces Genetics; John Innes Foundation: Norwich, UK, 2000.

13. Rebets, Y.; Ostash, B.; Luzhetskyy, A.; Hoffmeister, D.; Brana, A.; Mendez, C.; Salas, J.A.; Bechthold, A.; Fedorenko, V. Production of landomycins in Streptomyces globisporus 1912 and S. cyanogenus S136 is regulated by genes encoding putative transcriptional activators. FEMS Microbiol. Lett. 2003, 222, 149-153. [CrossRef]

14. Rodríguez Estévez, M.; Myronovskyi, M.; Gummerlich, N.; Nadmid, S.; Luzhetskyy, A. Heterologous Expression of the Nybomycin Gene Cluster from the Marine Strain Streptomyces albus subsp. chlorinus NRRL B-24108. Mar. Drugs 2018, 16, 435.

15. Mazodier, P.; Petter, R.; Thompson, C. Intergeneric conjugation between Escherichia coli and Streptomyces species. J. Bacteriol. 1989, 171, 3583-3585. [CrossRef] [PubMed] 
16. Myronovskyi, M.; Brötz, E.; Rosenkränzer, B.; Manderscheid, N.; Tokovenko, B.; Rebets, Y.; Luzhetskyy, A. Generation of new compounds through unbalanced transcription of landomycin A cluster. Appl. Microbiol. Biotechnol. 2016, 100, 9175-9186. [CrossRef] [PubMed]

17. Blin, K.; Medema, M.H.; Kottmann, R.; Lee, S.Y.; Weber, T. The antiSMASH database, a comprehensive database of microbial secondary metabolite biosynthetic gene clusters. Nucleic Acids Res. 2017, 45, D555-D559. [CrossRef]

18. Kearse, M.; Moir, R.; Wilson, A.; Stones-Havas, S.; Cheung, M.; Sturrock, S.; Buxton, S.; Cooper, A.; Markowitz, S.; Duran, C.; et al. Geneious Basic: An integrated and extendable desktop software platform for the organization and analysis of sequence data. Bioinformatics 2012, 28, 1647-1649. [CrossRef] [PubMed]

19. Myronovskyi, M.; Rosenkränzer, B.; Nadmid, S.; Pujic, P.; Normand, P.; Luzhetskyy, A. Generation of a cluster-free Streptomyces albus chassis strains for improved heterologous expression of secondary metabolite clusters. Metab. Eng. 2018, 49, 316-324. [CrossRef]

20. Rückert, C.; Albersmeier, A.; Busche, T.; Jaenicke, S.; Winkler, A.; Friðjónsson, Ó.H.; Hreggviðsson, G.Ó.; Lambert, C.; Badcock, D.; Bernaerts, K.; et al. Complete genome sequence of Streptomyces lividans TK24. J. Biotechnol. 2015, 199, 21-22. [CrossRef]

21. Siegl, T.; Tokovenko, B.; Myronovskyi, M.; Luzhetskyy, A. Design, construction and characterisation of a synthetic promoter library for fine-tuned gene expression in actinomycetes. Metab. Eng. 2013, 19, 98-106. [CrossRef]

22. Shimada, N.; Hasegawa, S.; Harada, T.; Tomisawa, T.; Fujii, A.; Takita, T. Oxetanocin, a novel nucleoside from bacteria. J. Antibiot. 1986, 39, 1623-1625. [CrossRef]

23. Morita, M.; Tomita, K.; Ishizawa, M.; Takagi, K.; Kawamura, F.; Takahashi, H.; Morino, T. Cloning of oxetanocin A biosynthetic and resistance genes that reside on a plasmid of Bacillus megaterium strain NK84-0128. Biosci. Biotechnol. Biochem. 1999, 63, 563-566. [CrossRef] [PubMed]

24. Bridwell-Rabb, J.; Zhong, A.; Sun, H.G.; Drennan, C.L.; Liu, H.-W. A B12-dependent radical SAM enzyme involved in oxetanocin A biosynthesis. Nature 2017, 544, 322-326. [CrossRef] [PubMed]

25. Bridwell-Rabb, J.; Kang, G.; Zhong, A.; Liu, H.-W.; Drennan, C.L. An HD domain phosphohydrolase active site tailored for oxetanocin-A biosynthesis. Proc. Natl. Acad. Sci. USA 2016, 113, 13750-13755. [CrossRef] [PubMed]

26. Grant, S.G.; Jessee, J.; Bloom, F.R.; Hanahan, D. Differential plasmid rescue from transgenic mouse DNAs into Escherichia coli methylation-restriction mutants. Proc. Natl. Acad. Sci. USA 1990, 87, 4645-4649. [CrossRef]

27. Flett, F.; Mersinias, V.; Smith, C.P. High efficiency intergeneric conjugal transfer of plasmid DNA from Escherichia coli to methyl DNA-restricting streptomycetes. FEMS Microbiol. Lett. 1997, 155, 223-229. [CrossRef]

(C) 2020 by the authors. Licensee MDPI, Basel, Switzerland. This article is an open access article distributed under the terms and conditions of the Creative Commons Attribution (CC BY) license (http://creativecommons.org/licenses/by/4.0/). 